\title{
Relative Gain Array and Singular Value Analysis to Improve the Control in a Biomass Pyrolysis Process*
}

\author{
Julian Hofmann ${ }^{1}$, Hans-Christian Holz ${ }^{2}$ and Lutz Gröll ${ }^{3}$
}

\begin{abstract}
The pairing of actuators and controlled variables for process control is non-trivial due to couplings among process quantities and further disturbing effects. Here, we consider the product gas stage in a biomass pyrolysis process. Based on a dynamic model of the latter, several analyses, namely Relative Gain Array (RGA), Dynamic Relative Gain Array (DRGA) and Singular Value Analysis (SVA) are performed to solve the pairing problem. In particular, we emphasize the practical benefit from DRGA's results by showing that challenges, like non-measurable disturbances or time delays, can be mitigated by simple changes in an implemented control concept.
\end{abstract}

\section{INTRODUCTION}

Most chemical processes are multivariable in nature. Hence, they are usually described by MIMO (multiple input multiple output) systems. To control such processes, a decentralised concept is often applied, i.e. the considered MIMO plant is controlled using a set of separately working single loop controls, which is also called multiloop control [1], [2]. This concept has proved its worth due to its simplicity and has been extended for several applications [3], [4]. However, the performance essentially depends on a suitable pairing of controlled and manipulated variables [5]. Often the pairing is realized intuitively [6], but more straight forward methods are known. By introducing RGA, Bristol [7] developed a concept to quantify and evaluate the static interaction between two SISO control loops. Its extension to an arbitrary finite number of SISO control loops as well as to the dynamic case is described in [8], [6], [9], [1] and is often referred to as DRGA. Another concept to investigate multivariable control problems is SVA [10], [1], [11], which is based on singular value decomposition (SVD). Some other papers present further developments w.r.t the RGA method, either by expanding its application area to robustness analysis [12], model uncertainty [13] or by creating RGA based methods and therefore introduce new interaction measures (IMs), like the Average Relative Gain Matrix (ARGM) [14], the Hankel Interaction Index (HII) [15], the Effective Relative Gain Array (ERGA) [16]

\footnotetext{
*This work was not supported by any organization

${ }^{1}$ Julian Hofmann is with the Institute for Automation and applied Informatics, Karlruhe Institute of Technology, Hermann-von-Helmholtz-Platz 1, 76344 Eggenstein-Leopoldshafen, Germany julian.hofmannekit.edu

${ }^{2} \mathrm{Hans}$ Christian Holz is with the Institute for Automation and applied Informatics, Karlruhe Institute of Technology, Hermann-von-HelmholtzPlatz 1, 76344 Eggenstein-Leopoldshafen, Germany

uaeuv@student.kit.edu

${ }^{3}$ Lutz Gröll is with the Institute for Automation and applied Informatics, Karlruhe Institute of Technology, Hermann-von-Helmholtz-Platz 1, 76344 Eggenstein-Leopoldshafen, Germany

lutz.groellekit.edu
}

or the state-Dynamic Relative Gain Array (s-DRGA) [17] Another earlier field of research regarding RGA has derived approximate analytical expressions [18], [19] or numerical approaches [20] for Bristol's (static) RGA by considering dual composition control control. Additionally, there exist numerous other publications dealing with the application of RGA [21], [22], [23] and SVA [24] in the context of multivariable control.

However, a huge part of them presents rather academic examples without testing the results of the analysis in a validated simulation model of a real plant. Furthermore, DRGA is rarely considered in practical applications [25], [6]. As DRGA provides suitable pairings for different frequency domains of the plant model, it remains unanswered in the literature, which effects influence the operation frequency domains of a real plant. To fill this gap, we firstly give an overview, how different frequency domains can be excited in process plants. Secondly, we show for our application that the time delays of the actuators essentially influence the operation frequency domain. Therefore, we give an idea, how to identify a relation between plant disturbances and excited frequency domains.

This paper is organized as follows. In Sec. II, we present a dynamic model of the product gas stage in a biomass pyrolysis process. After introducing control nomenclature for the physical variables, the model is classified as nonlinear differential algebraic equation (DAE) system with constant input delay in semi-explicit form. Via linearization about two characteristic operation points (OPs) neglecting input delays, quantisation and saturation effects, a transfer function matrix for each operation point is obtained, s. Sec. III-A. In Sec. III$\mathrm{B}$, III-C and III-D, the plant is analyzed by applying RGA, DRGA and SVA, where corresponding results are further discussed. Finally in Sec. IV, the recommendations to modify the control concept are tested and verified in simulations.

\section{MODELING}

In this section, we shortly describe the considered process and give an overview of relevant model equations.

\section{A. Description of the Product Gas Stage}

The Karlsruhe Institute of Technology (KIT) tests the production of synthetic fuel from renewable raw materials in the pilot plant bioliq $\AA$, which consists of four partial plants. The partial plant bioliq I, which is modelled in the next section, is used to produce an organic condensate of high energy density from dried, finely chopped biomass, e.g. straw. This charge material is introduced into a reactor, 
where the pyrolysis reaction takes place. The intermediate products consist of different gases and vapour, while all solid parts like coke and sand are separated. Subsequently, all gases and vapour pass a cooler and two condensers, which separate the liquid phase from the vapour, such that the combustible product gas is obtained. While the condensate acts as charge material of the downstream process step, the product gas is burnt together with atmospheric oxygen in an excess gas burner and is then dissipated to the ambience. In order to transport the gases and vapour from the reactor to the excess gas burner, a blower provides the desired pressure ratios. When conveying a sufficiently high flow rate, a safe operation of the blower is ensured. This is realized by branching off a part of the product gas in front of the burner and feeding it back to the condensers.

\section{B. Dynamic Model of the Product Gas Stage}

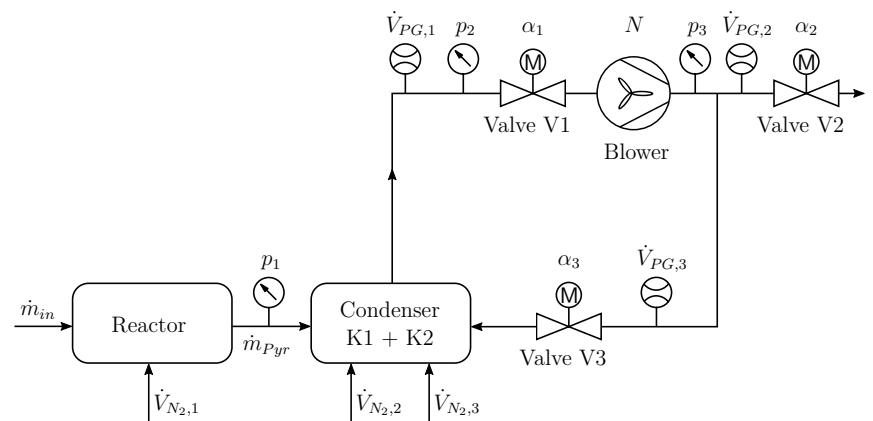

Fig. 1: Flow sheet of the product gas stage in the considered biomass pyrolysis process

The physical model of the product gas stage in the considered biomass pyrolysis process is described by the flow sheet in Fig. 1. In the following, the model equations are given and shortly explained (s. [26], [27], [28], [29] for more details). The nomenclature for physical variables, parameters and constants is shown in TABLE I.

1) Pyrolysis reaction: Since a detailed model of the complex pyrolysis reaction is impractical from a control engineering perspective, it is simplified as PT1 element

$$
\frac{\mathrm{d}}{\mathrm{d} t} \dot{m}_{P y r}=\frac{1}{T_{P y r}}\left(k_{P y r} \dot{m}_{i n}-\dot{m}_{P y r}\right) .
$$

2) Flow Balance: By balancing the product gas volume flows, the relation

$$
\dot{V}_{P G, 1}-\dot{V}_{P G, 2}-\dot{V}_{P G, 3}=0
$$

is obtained.

3) Gas mixture: Assume that the product gas is a perfect mixture (Law of Adamat) of pyrolysis and nitrogen gas. The density of the product gas is then given by

$$
\rho_{P G}=\frac{\rho_{N_{2}} \dot{V}_{N_{2}, \text { res }}+\dot{m}_{P y r}}{\dot{V}_{N_{2}, \text { res }}+\frac{\dot{m}_{P y r}}{\rho_{P y r}}} .
$$

4) Plant pressure: If isothermal state changes and slow pressure changes are assumed, the plant pressure can be modelled as integrator over incoming and outgoing mass flows [27], i.e.

$$
\frac{\mathrm{d}}{\mathrm{d} t} p_{1}=\frac{1}{T_{p_{1}}}\left(\dot{m}_{P y r}+\rho_{N_{2}} \dot{V}_{N_{2}, r e s}-\rho_{P G} \dot{V}_{P G, 2}\right) .
$$

5) Plant flow resistance: In order to describe the behaviour of the plant flow resistance, the simple approach $\Delta p=$ $c \dot{V}^{2}$ is applied and therefore, it follows that

$$
p_{1}-p_{2}=c_{P l} \dot{m}_{P y r}^{2} .
$$

6) Blower and valves: While the blower is represented by its static characteristic curve, the valves have equalpercentage characteristics. Since the blower and valve V1 are serially connected, the pressure difference over these two elements yields

$$
\begin{aligned}
p_{3}-p_{2}= & \left(k_{g} g_{2}-\left(c_{V 1}+\frac{\exp \left(-2 a_{s, V 1} \widetilde{\alpha}_{1}\right)}{a_{1, V 1}^{2}}\right)\right) \dot{V}_{P G, 1}^{2} \\
& +k_{g} g_{1} N_{n} \widetilde{N} \dot{V}_{P G, 1}+k_{g} g_{0}\left(N_{n} \widetilde{N}\right)^{2} .
\end{aligned}
$$

By connecting valve $\mathrm{V} 2$ to the ambience, its normalized after-pressure is zero, and can be described by

$$
p_{3}=\left(c_{V 2}+\frac{\exp \left(-2 a_{s, V 2}\left(\widetilde{\alpha}_{2}-\alpha_{2, \min }\right)\right)}{a_{1, V 2}^{2}}\right) \dot{V}_{P G, 2}^{2}
$$

within its effective operation range represented by $\alpha_{2, \min }$. Finally, the equation for valve V3 is simply given by its equal-percentage characteristic, such that

$$
p_{3}-p_{2}=\left(c_{V 3}+\frac{\exp \left(-2 a_{s, V 3} \widetilde{\alpha}_{3}\right)}{a_{1, V 3}^{2}}\right) \dot{V}_{P G, 3}^{2} .
$$

7) Blower drive: The blower drive consists of a speedregulated electric motor, which transforms the desired rotational speed $N$ into the effective one $\widetilde{N}$. This process is modelled by a PT1 element with rise limitation, i.e.

$$
\frac{\mathrm{d}}{\mathrm{d} t} \widetilde{N}=\operatorname{sat}_{l_{-, N}}^{l_{+, N}}\left(k_{N}(N-\widetilde{N})\right),
$$

where the saturation of a signal $x$ with limits $l_{-}$and $l_{+}$is defined by

$$
\operatorname{sat}_{l_{-}}^{l_{+}}(x)= \begin{cases}l_{-}, & x<l_{-} \\ x, & l_{-} \leq x \leq l_{+} \quad, \quad l_{+}>l_{-} . \\ l_{+}, & x>l_{+}\end{cases}
$$

8) Valve actuators: Closely related to (9) are the equations for the valve actuators. Besides rising limitations, quantisation effects and input delays are additionally included, such that

$$
\frac{\mathrm{d}}{\mathrm{d} t} \widetilde{\alpha}_{i}(t)=\operatorname{sat}_{l_{-, \alpha_{i}}}^{l_{+, \alpha_{i}}}\left(k_{\alpha_{i}}\left(\text { quant }_{q_{\alpha_{i}}}\left(\alpha_{i}\left(t-T_{d, \alpha_{i}}\right)\right)-\widetilde{\alpha}_{i}(t)\right)\right)
$$

is obtained. Here, the indices $i=1,2,3$ represent the corresponding valve. Furthermore, the operator quant $_{q}(x)$ denotes the quantisation of the signal $x$ with step size $q$ and is defined by

$$
\text { quant }_{q}(x)=\operatorname{sgn}(x) \cdot q \cdot\left\lfloor\frac{|x|}{q}+\frac{1}{2}\right\rfloor, \quad q>0 .
$$




\begin{tabular}{|c|c|c|c|c|c|c|c|c|c|}
\hline \multicolumn{10}{|c|}{ Variables } \\
\hline \multicolumn{4}{|c|}{ Description } & Unit & \multicolumn{2}{|c|}{ Sym. } & \multicolumn{2}{|l|}{ Description } & Unit \\
\hline & $\begin{array}{l}\widetilde{\alpha}_{i} \\
\alpha_{i} \\
\dot{m}_{i n} \\
\dot{m}_{P y r} \\
\widetilde{N} \\
N \\
p_{1} \\
p_{2}\end{array}$ & $\begin{array}{l}\text { effective position of valve } \mathrm{V} i \\
\text { desired position of valve } \mathrm{V} i \\
\text { charge mass flow into reactor } \\
\text { pyrolysis gas mass flow into re } \\
\text { normalized effective roational } \\
\text { normalized desired roational sp } \\
\text { normalized pressure in pressure } \\
\text { normalized pressure in front of }\end{array}$ & $\begin{array}{l}\text { or } \\
\text { d of blower } \\
\text { of blower } \\
\text { servoir } \\
\text { lve V1 }\end{array}$ & $\begin{array}{l}- \\
- \\
\frac{\mathrm{kg}^{3}}{\mathrm{~s}} \\
\frac{\mathrm{kg}^{3}}{\mathrm{~s}} \\
- \\
- \\
\text { mbar } \\
\text { mbar }\end{array}$ & & $\begin{array}{l}p_{3} \\
\rho_{P G} \\
\dot{V}_{N_{2}, \text { res }}\end{array}$ & \multicolumn{2}{|c|}{$\begin{array}{l}\text { normalized pressure after blower } \\
\text { std. density of product gas } \\
\text { resultant std. volume flow of nitrogen gas: } \\
\dot{V}_{N_{2}, \text { res }}=\dot{V}_{N_{2}, 1}+\dot{V}_{N_{2}, 2}+\dot{V}_{N_{2}, 3} \\
\text { std. volume flow of product gas through blower } \\
\text { std. volume flow of product gas to gas burner } \\
\text { std. volume flow of product gas in feedback }\end{array}$} & $\begin{array}{l}\mathrm{mbar} \\
\frac{\mathrm{kg}^{3}}{\mathrm{~m}^{3}} \\
\frac{\mathrm{m}^{3}}{\mathrm{~h}} \\
\frac{\mathrm{m}^{3}}{\mathrm{~h}} \\
\frac{\mathrm{m}^{3}}{\mathrm{~h}} \\
\frac{\mathrm{m}^{3}}{\mathrm{~h}}\end{array}$ \\
\hline \multicolumn{10}{|c|}{ Parameters and Constants } \\
\hline Sym. & \multicolumn{2}{|c|}{ Description } & Values & Unit & Sym. & \multicolumn{2}{|c|}{ Description } & Values & Unit \\
\hline $\begin{array}{l}a_{1, V i} \\
a_{s, V i} \\
\alpha_{2, \min } \\
c_{P l} \\
c_{V i} \\
g_{0} \\
g_{1} \\
g_{2} \\
k_{g} \\
k_{N}\end{array}$ & \multicolumn{2}{|c|}{$\begin{array}{l}\text { valve } \mathrm{V} i \text { characteristic curve parameter } \\
\text { valve } \mathrm{V} i \text { characteristic curve parameter } \\
\text { lower valve } \mathrm{V} 2 \text { actuator limit } \\
\text { flow resistance of front plant } \\
\text { flow resistance of pipe to valve } \mathrm{V} i \\
\text { blower characteristic curve parameter } \\
\text { blower characteristic curve parameter } \\
\text { blower characteristic curve parameter } \\
\text { proportionality factor of blower } \\
\text { gain factor of blower drive }\end{array}$} & $\begin{array}{l}11.8 ; 0.5 ; 0.1 \\
3.9 ; 6.8 ; 5.9 \\
0.22 \\
0.5 \cdot 10^{-3} \\
0 ; 0.01 ; 0.006 \\
1.0 \cdot 10^{-5} \\
5.2 \cdot 10^{-5} \\
-9.7 \cdot 10^{-4} \\
0.9 \\
10\end{array}$ & $\begin{array}{l}\frac{\mathrm{m}^{3}}{\mathrm{~h} \sqrt{\mathrm{mbar}}} \\
- \\
- \\
\frac{\mathrm{h}^{2} \mathrm{mbar}}{\mathrm{m}^{6}} \\
\frac{\mathrm{h}^{2} \mathrm{mbar}}{\mathrm{m}^{6}} \\
\frac{\mathrm{h}^{2} \mathrm{mbar}}{\mathrm{m}^{6}} \\
\frac{\mathrm{minhmar}^{\mathrm{m}}}{\mathrm{m}^{3}} \\
\min ^{2} \mathrm{mbar} \\
- \\
\frac{1}{\mathrm{~s}}\end{array}$ & $\begin{array}{l}k_{P y r} \\
k_{\alpha_{i}} \\
l_{ \pm, N} \\
l_{ \pm, \alpha_{i}} \\
N_{n} \\
q_{\alpha_{i}} \\
\rho_{N_{2}} \\
\rho_{P y r} \\
T_{p_{1}} \\
T_{P y r} \\
T_{d, \alpha_{i}}\end{array}$ & \multicolumn{2}{|c|}{$\begin{array}{l}\text { reaction factor of pyrolysis } \\
\text { gain factor of valve } \mathrm{V} i \text { actuator } \\
\text { slope limit of blower drive } \\
\text { slope limit of valve } \mathrm{V} i \text { actuator } \\
\text { blower nominal rotation speed } \\
\text { valve } i \text { actuator quant step size } \\
\text { std. density of nitrogen gas } \\
\text { std. density of pyrolysis gas } \\
\text { pressure reservoir time constant } \\
\text { time constant of pyrolysis } \\
\text { time delay of valve Vi actuator }\end{array}$} & $\begin{array}{l}0.2 \\
2.0 ; 1.5 ; 1.0 \\
\pm \frac{1}{60} \\
\pm\{0.3 ; 0.04 ; 0.01\} \\
6544.4 \\
0.03 ; 0.03 ; 0.01 \\
1.25 \\
1.6 \\
32.718 \\
30 \\
1.5 ; 2.5 ; 1.5\end{array}$ & $\begin{array}{l}- \\
\frac{1}{\mathrm{~s}} \\
\frac{1}{\mathrm{~s}} \\
\frac{1}{\mathrm{~s}} \\
\frac{1}{\mathrm{~min}} \\
- \\
\frac{\mathrm{kg}}{\mathrm{m}^{3}} \\
\frac{\mathrm{kg}}{\mathrm{m}^{3}} \\
\mathrm{~s} \\
\mathrm{~s} \\
\mathrm{~s}\end{array}$ \\
\hline
\end{tabular}

TABLE I: Nomenclature for physical variables, parameters and constants

Hereinafter, (1) to (10) are referred to as plant and include the process as well as the actuator model. All parameters are identified using output error least squares, i.e. the output error $e_{j}=\hat{y}_{j}-y_{j}, j=1, \ldots, n$ of the measured output $y_{j}$ and the simulated one $\hat{y}_{j}$ is minimized by

$$
\min _{\hat{\theta}} \sum_{j=1}^{n} e_{j}^{2}
$$

where $\hat{\theta}$ denotes the vector of estimated parameters. Since the parameter identification is not the main focus of this paper, it is not further discussed.

\section{ANALYSIS AND DISCUSSION}

In the following, the plant is represented as a DAE system, which is linearized about two operating points and transfer matrices for each one are obtained. The methodologies of RGA, DRGA and SVA are firstly described, secondly applied to the plant and thirdly corresponding results are discussed.

\section{A. Plant Simplification}

At first, the physical variables are renamed into control variables, such that

$$
\begin{aligned}
u & =\left[\begin{array}{llllll}
u_{1} & u_{2} & u_{3} & u_{4} & z_{1} & z_{2}
\end{array}\right]^{\top} \\
& :=\left[\begin{array}{llllll}
\alpha_{1} & \alpha_{2} & \alpha_{3} & N & \dot{m}_{i n} & \dot{V}_{N_{2}, r e s}
\end{array}\right]^{\top}, \\
x & =\left[\begin{array}{llllll}
x_{1} & x_{2} & x_{3} & x_{4} & x_{5} & x_{6}
\end{array}\right]^{\top} \\
& :=\left[\begin{array}{llllll}
p_{1} & \dot{m}_{P y r} & \widetilde{\alpha}_{1} & \widetilde{\alpha}_{2} & \widetilde{\alpha}_{3} & \widetilde{N}
\end{array}\right]^{\top}, \\
d & =d_{1}:=p_{3}, \\
y & =\left[\begin{array}{lll}
y_{1} & y_{2} & y_{3}
\end{array}\right]^{\top} \\
& :=\left[\begin{array}{lll}
p_{1} & p_{3} & \dot{V}_{P G, 1}
\end{array}\right]^{\top} .
\end{aligned}
$$

Subsequently, the plant in control nomenclature is rewritten as nonlinear semi-explicit delayed DAE system with constant input delays

$$
\begin{aligned}
& \dot{x}(t)=f\left(x(t), d(t), u(t), u\left(t-T_{d, \alpha_{i}}\right)\right), \\
& x(0)=x_{0}, u_{0}(\theta)=\phi(\theta), \theta \in\left[-T_{d, \alpha_{i}}, 0\right], \\
& 0=g(x(t), d(t), u(t)), \\
& y(t)=h(x(t), d(t), u(t))
\end{aligned}
$$

with $f: \mathbb{R}^{6} \times \mathbb{R} \times \mathbb{R}^{6} \rightarrow \mathbb{R}^{6}, g: \mathbb{R}^{6} \times \mathbb{R} \times \mathbb{R}^{6} \rightarrow \mathbb{R}$ and $h$ : $\mathbb{R}^{6} \times \mathbb{R} \times \mathbb{R}^{6} \rightarrow \mathbb{R}^{3}$. Note that the physical variables $p_{2}, \dot{V}_{P G, 2}$ and $\dot{V}_{P G, 3}$ are eliminated when transforming (1) to (10) into (13) and therefore neither appear in the control nomenclature (12) nor in the plant's DAE realization (13). For the sake of linearization, input delays, quantisations and saturations are neglected, i.e.

$$
\begin{aligned}
u\left(t-T_{d, \alpha_{i}}\right) & \approx u(t), \\
\operatorname{quant}_{q}(u(t)) & \approx u(t), \\
\operatorname{sat}_{l_{-}}^{l_{+}}(u(t)) & \approx u(t)
\end{aligned}
$$

is assumed in (13). Although the latter effects are neglected to simplify further calculations, they still exist in the plant (13). As all further analysis results are tested using (13), it will turn that they remain valid despite this simplification, s. Sec. IV.

Since $\frac{\partial g}{\partial d^{\top}}$ in (13) assuming (14) is non-singular, (13) is called index 1 DAE system and therefore the linearization can be used for further analysis [30]. Then, the linearized 
DAE system is described by

$$
\begin{aligned}
& \Delta \dot{x}(t)=\left.\frac{\partial f}{\partial x^{\top}}\right|_{\mathrm{OP}} \Delta x(t)+\left.\frac{\partial f}{\partial d^{\top}}\right|_{\mathrm{OP}} \Delta d(t)+\left.\frac{\partial f}{\partial u^{\top}}\right|_{\mathrm{OP}} \Delta u(t), \\
& \Delta x\left(0^{-}\right)=x_{0}-x_{e}, \\
& 0=\left.\frac{\partial g}{\partial x^{\top}}\right|_{\mathrm{OP}} \Delta x(t)+\left.\frac{\partial g}{\partial d^{\top}}\right|_{\mathrm{OP}} \Delta d(t)+\left.\frac{\partial g}{\partial u^{\top}}\right|_{\mathrm{OP}} \Delta u(t), \\
& \Delta y(t)=\left.\frac{\partial h}{\partial x^{\top}}\right|_{\mathrm{OP}} \Delta x(t)+\left.\frac{\partial h}{\partial d^{\top}}\right|_{\mathrm{OP}} \Delta d(t)+\left.\frac{\partial h}{\partial u^{\top}}\right|_{\mathrm{OP}} \Delta u(t) .
\end{aligned}
$$

Solving (15c) w.r.t. $\Delta d$ leads to

$$
\begin{aligned}
\Delta d(t) & =\left.\left(-\left(\frac{\partial g}{\partial d^{\top}}\right)^{-1} \frac{\partial g}{\partial x^{\top}}\right)\right|_{\mathrm{OP}} \Delta x(t) \\
& +\left.\left(-\left(\frac{\partial g}{\partial d^{\top}}\right)^{-1} \frac{\partial g}{\partial u^{\top}}\right)\right|_{\mathrm{OP}} \Delta u(t) .
\end{aligned}
$$

By inserting (16) into (15a) and (15d), an index-reduction is performed and the corresponding LTI state space realization

$$
\begin{aligned}
& \Delta \dot{x}(t)=A \Delta x(t)+B \Delta u(t), \quad \Delta x\left(0^{-}\right)=x_{0}-x_{e}, \\
& \Delta y(t)=C \Delta x(t)+D \Delta u(t)
\end{aligned}
$$

is obtained. Appropriate to the extension of the HartmanGrobman-Theorem [30], the nonlinear index 1 DAE system has the same local behaviour as the linearized one, iff the corresponding equilibrium is hyperbolic. The latter statement can directly be read from the diagonal elements of

$$
A=\left[\begin{array}{cccccc}
\square & \square & \square & \square & \square & \square \\
0 & -\frac{1}{T_{P y r}} & 0 & 0 & 0 & 0 \\
0 & 0 & -k_{\alpha_{1}} & 0 & 0 & 0 \\
0 & 0 & 0 & -k_{\alpha_{2}} & 0 & 0 \\
0 & 0 & 0 & 0 & -k_{\alpha_{3}} & 0 \\
0 & 0 & 0 & 0 & 0 & -k_{N}
\end{array}\right],
$$

where $\square$ abbreviates large formulas. The calculation of $A_{11}$ is tedious, but for all possible equilibria we get a negative value due to the stability of the linearized model.

By applying the Laplace transformation to (17), the transfer function matrix for the corresponding OP is

$$
G(s)=C\left(s I_{n}-A\right)^{-1} B+D ; \quad G(s) \in \mathbb{R}[s]^{3 \times 6} .
$$

This transfer function matrix is analyzed via RGA, DRGA and SVA in the next section.

\section{B. $R G A$}

1) RGA Description: Originally, Bristol [7] introduced RGA as follows

$$
[\Lambda]_{i j}=\lambda_{i j}=\frac{k_{i j}}{\widetilde{k}_{i j}}=\frac{\left(\frac{\partial y_{i}}{\partial u_{j}}\right)_{u_{k}=0, k \neq j}}{\left(\frac{\partial y_{i}}{\partial u_{j}}\right)_{y_{k}=0, k \neq i}},
$$

where $k_{i j}$ denotes the open loop gain and $\widetilde{k}_{i j}$ the closed loop gain. The relative gain $\lambda_{i j}$ represents an interaction measure for the effect of a manipulated variable $u_{j}$ on a controlled variable $y_{i}$. While $k_{i j}$ simply corresponds to an element of the static gain matrix

$$
K=G(0),
$$

the determination of $\widetilde{k}_{i j}$, which corresponds to the effect of a manipulated variable $u_{j}$ on a controlled variable $y_{i}$ when all other controlled variables are constant, is more involved and often done experimentally. Nevertheless, the relative gain array $\Lambda$ can equivalently be calculated, s. e.g. [9], via

$$
\Lambda=K \circ\left(K^{-1}\right)^{\top}
$$

where $\circ$ is the Hadamard product.

Subsequently, five paring rules [1] based on the RGA's results are shortly recalled:

1) $\lambda_{i j}=1$ : Open and closed loop gain are equal. Optimal preconditions to control $y_{i}$ with $u_{j}$.

2) $\lambda_{i j}=0$ : Either open loop gain is zero or closed loop gain is infinitely large. Controlling $y_{i}$ with $u_{j}$ is not recommended.

3) $0<\lambda_{i j}<1$ : The closed loop gain is bigger than the open loop one. The smaller $\lambda_{i j}$ is, the less suitable is $u_{j}$ to control $y_{i}$.

4) $1<\lambda_{i j}<\infty$ : The closed loop gain is bigger than the open loop one. Controlling $y_{i}$ with $u_{j}$ is however possible.

5) $\lambda_{i j}<0$ : Closed and open loop gain have opposite signs. Controlling $y_{i}$ with $u_{j}$ is not recommended.

Furthermore, note that the sum of the elements in $\Lambda$ in each line or column is one. All elements are dimensionless and invariant under scaling [7]. The controllability of the considered system gets lost for $\left|\lambda_{i j}\right| \rightarrow \infty$ [9].

2) RGA applied to the Plant: While $u_{1}, u_{2}$ and $u_{3}$ are manipulated variables, $u_{4}$ is a steering variable and $z_{1}$ as well as $z_{2}$ are disturbance variables. Therefore, $K$ is split into an effective part $K_{e}$ and a residual part $K_{r}$, i.e.

$$
K=\left[\begin{array}{ll}
K_{e} & K_{r}
\end{array}\right]
$$

such that

$$
\left[\begin{array}{l}
\Delta y_{1, s} \\
\Delta y_{2, s} \\
\Delta y_{3, s}
\end{array}\right]=K_{e}\left[\begin{array}{l}
\Delta u_{1, s} \\
\Delta u_{2, s} \\
\Delta u_{3, s}
\end{array}\right]+K_{r}\left[\begin{array}{l}
\Delta u_{4, s} \\
\Delta z_{1, s} \\
\Delta z_{2, s}
\end{array}\right],
$$

where the index $s$ denotes the static case. Substituting $K$ by $K_{e}$ in (22), gives

$$
\Lambda_{1}=\left[\begin{array}{ccc}
0.7650 & 0 & 0.2350 \\
0 & 1 & 0 \\
0.2350 & 0 & 0.7650
\end{array}\right]
$$

for operation point 1 , and

$$
\Lambda_{2}=\left[\begin{array}{ccc}
0.9209 & 0 & 0.0791 \\
0 & 1 & 0 \\
0.0791 & 0 & 0.9209
\end{array}\right]
$$

for operation point 2 .

3) RGA Discussion: The resulting pairing is $u_{1} \leftrightarrow y_{1}$, $u_{2} \leftrightarrow y_{2}$ and $u_{3} \leftrightarrow y_{3}$. This result corresponds to the currently implemented control concept. 


\section{C. $D R G A$}

1) DRGA Description: Expanding RGA (22) to the dynamic case [9] intuitively leads to DRGA defined by

$$
\Lambda_{d}(s)=G(s) \circ\left(G(s)^{-1}\right)^{\top} .
$$

According to the expanded pairing rule, $\lambda_{i j}$ of pairs of manipulated and controlled variable should be approximately one for frequencies within the closed loop's bandwidth. However, because of several disturbances, it is often hard to analyse or to determine a fixed bandwidth for the frequencies of a closed loop. Thus, it is reasonable to consider a large frequency bandwidth in the framework of the analysis.

2) DRGA applied to the Plant: In order to apply the DRGA to the plant, $G(s)$ is split in the same way as $K$ in (23) and the effective part $G_{e}(s)$ is used in (27). The results for both OPs are illustrated in Fig. 2, where it can be seen that the pairing changes for $\omega \gtrsim 10^{-2} \frac{1}{\mathrm{~s}}$ compared to the static case. In this frequency domain, the pairing $u_{1} \leftrightarrow y_{2}, u_{2} \leftrightarrow y_{1}$ and $u_{3} \leftrightarrow y_{3}$ should be chosen.

3) DRGA Discussion: Referring to the lack of motivation for DRGA from a practical perspective, we will firstly explain some aspects. The obvious motivation to consider a wide frequency bandwidth is given by periodic reference signals. However, this does not hold for process plants, since most control tasks consist of set point controller design. Nevertheless, there are other reasons to investigate a wide frequency domain for process engineering applications. For instance, it is possible that a certain partial transfer function damps lower frequencies and excites higher ones (or vice versa), which can be interpreted as internal disturbance. Hence, a pair must be chosen, such that the disturbance effect can quickly be detected at the plant output, enabling the controller to react. In the following situations, an excitation of different frequency domains in a plant or in a control loop can generally appear:

1) external disturbances, e.g. day/night phases, temperature variations, wind, pressure or voltage fluctuations,

2) plant input disturbances, e.g. changes in the input properties (concentration, viscosity, mass flow),

3) plant internal disturbances, e.g.

- forced nonlinearities due to quantisations and switching operations,

- structural disturbances due to neglected couplings and nonlinear terms,

- time discrete realizations in a continuous process,

- time delays in the process

4) periodic reference signals with different frequencies (typical in mechanical or electrical applications)

For most of the above mentioned aspects, there exist conventional control methods, e.g. periodic reference signals and measurable disturbances can be controlled via feedforward. Hence, the results of DRGA can be used to fight non-modeled or non-measurable disturbances by letting the controller overwhelm them.

Reconsidering our application, a reason for investigating a higher frequency domain and thus changing the pairing lies in the time delays of the valve actuators. In order to motivate the latter statement, consider the transfer function of a time delay element with delay $T_{d}$ under feedback, i.e.

$$
T(s)=\frac{\mathrm{e}^{-s T_{d}}}{1+\mathrm{e}^{-s T_{d}}} .
$$

Its poles are calculated by using the approach $s_{0}=\sigma+\mathrm{i} \omega$. A separation of real and imaginary part gives

$$
\begin{aligned}
1+\mathrm{e}^{-\sigma T_{d}} \cos \omega T_{d} & =0, \\
-\mathrm{e}^{-\sigma T_{d}} \sin \omega T_{d} & =0 .
\end{aligned}
$$

From (29b), it follows $\omega=\frac{k \pi}{T_{d}}, k \in \mathbb{Z}$, such that (29a) is fulfilled for $k=2 \widetilde{k}+1$ and therefore

$$
\omega=\frac{(2 \widetilde{k}+1) \pi}{T_{d}}, \quad \widetilde{k} \in \mathbb{Z} .
$$

For $\widetilde{k}=0$, the frequency $\omega=\frac{\pi}{T_{d}}$ is obtained. Since $T_{d} \approx 2 \mathrm{~s}$ is valid for the valve actuators' delays, frequencies $\omega \approx 10^{0} \frac{1}{\mathrm{~s}}$ can be excited.

To conclude, by investigating mathematical models of different disturbances, a relation between them and the excitation of corresponding frequency domains can be given.

D. SVA

1) SVA Description: Another possibility to analyse multivariable control problems is SVA. The singular values $\sigma_{i} \in \mathbb{R}_{0}^{+}, i=1 \ldots m$ of a matrix $M \in \mathbb{C}^{n \times m}$ are defined by

$$
\sigma_{i}(M)=\sqrt{\lambda_{i}\left\{M^{H} M\right\}},
$$

where $\lambda_{i}$ are the eigenvalues of the symmetrical matrix $M^{H} M$ and $M^{H}$ denotes the conjugate transpose. If the singular values are arranged as follows

$$
\begin{aligned}
\sigma_{1}(M) \geq \sigma_{2}(M) & \geq \ldots \geq \sigma_{k} M>0, \\
\sigma_{k+1}(M)=\sigma_{k+2}(M) & =\ldots=\sigma_{m}(M)=0,
\end{aligned}
$$

the first $k=\operatorname{rk}\left(M^{H} M\right)$ singular values are positive, the remaining ones are zero [10]. Then, the condition $\kappa \in[1, \infty)$, s. e.g. [1], of $M$ can be introduced by

$$
\kappa(M)=\frac{\sigma_{1}}{\sigma_{k}}
$$

for non-singular $M$. If $\kappa$ is large, $M$ is called badly conditioned, which is an evidence for singularity and bad invertibility. In order to perform an SVA based on $\kappa(K)$, where $K$ is the static transfer matrix, s. (21), the following remarks should be considered [1]:

1) The number of manipulated and controlled variables must be the same when $\kappa(K)$ is analysed.

2) If $\kappa(K)$ is large, the system is badly conditioned and thus hard to control, independent of the RGA's result.

3) The absolute value of $\kappa(K)$ depends on the scaling of manipulated and controlled variables.

Since no measure can be given to declare $\kappa(K)$ as large due to differently scaled variables, a comparison by pairwise cancellation of manipulated and controlled variables is nevertheless possible. In this context, configurations (configs) 

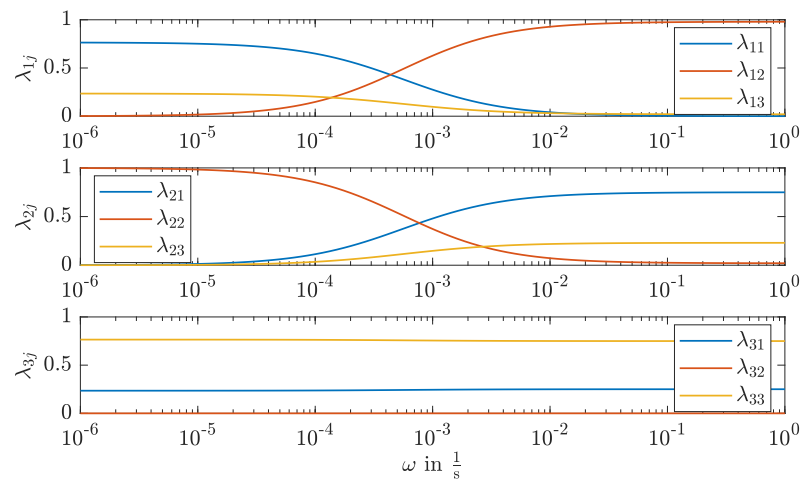

(a) Operation point 1

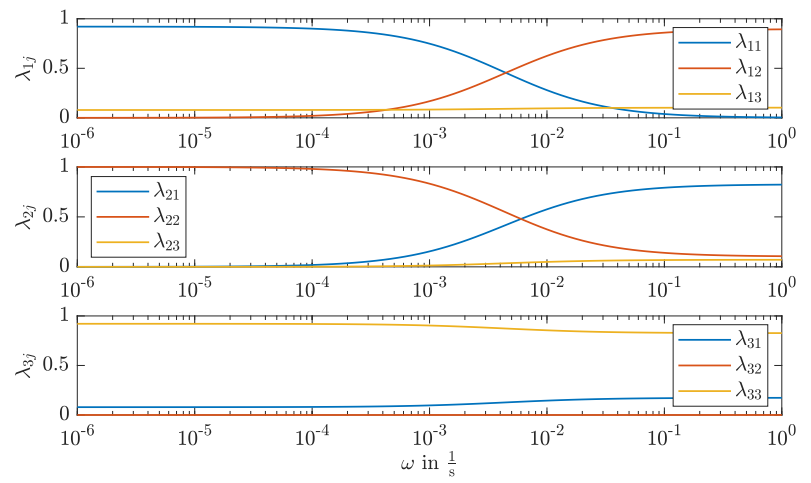

(b) Operation point 2

Fig. 2: DRGA for both operation points

with lower conditions than the original one are preferred. Moreover, 3) illustrates an important difference to RGA, where the result does not depend on the scaling.

2) SVA applied to the Plant: Using the matrix $K_{e}$, which is defined via (23) and (24), the condition is

$$
\kappa\left(K_{e}\right):=\kappa\left(K_{e, 0}\right)=19.01
$$

for operation point 1 and

$$
\kappa\left(K_{e}\right):=\kappa\left(K_{e, 0}\right)=20.23
$$

for operation point 2, where the second index denotes the corresponding config. However, these two absolute values cannot be interpreted, since $u_{i}(t)$ and $y_{i}(t), i=1,2,3$, are not scaled equivalently, s. also 3).

3) SVA Discussion: Nevertheless, an interpretation is possible by systematically cancelling pairs of manipulated and controlled variables. The result of the latter approach is shown in TABLE II, which allows the following conclusions:

- Configs 2 and 8 indicate a badly conditioned $K_{e}$ and are thus practically infeasible.

- Since the conditions of configs 3,4 and 7 have not decreased compared to config 0 , they can be neglected.

- Although configs 5, 6 and 9 have lower conditions than config 0 , they are infeasible from process engineering perspective.

- Config 1 has a lower condition than config 0 and practically means that the third control loop is omitted, which is possible from process engineering perspective.

Hence, config 1 is the only feasible cancellation (control loop reduction), since deviations from the desired value are allowed for $y_{3}$.

Apart from systematic cancelling of pairs, it is also conceivable to investigate the replacement of $u_{1}, u_{2}$, and $u_{3}$ by $u_{4}$, since the latter one is steered, while the others are controlled. The corresponding result is depicted in TABLE III, which indicates that replacing $u_{1}$ by $u_{4}$ is the only possible alternative compared to the original config 0 . Practically, this result means that the pressure difference along the blower is controlled by the blower's rotational speed instead of the position of valve V1, which is feasible from process

\begin{tabular}{|c|c|c|c|c|}
\hline config & controlled & manipulated & \multicolumn{2}{|c|}{$\kappa\left(K_{e, n}\right), n=0,1, \ldots, 9$} \\
& variables & variables & OP 1 & OP 2 \\
\hline 0 & $y_{1}, y_{2}, y_{3}$ & $u_{1}, u_{2}, u_{3}$ & 19.01 & 20.23 \\
\hline 1 & $y_{1}, y_{2}$ & $u_{1}, u_{2}$ & 13.28 & 13.06 \\
2 & $y_{1}, y_{2}$ & $u_{1}, u_{3}$ & $1.4 \mathrm{e}+16$ & $8.1 \mathrm{e}+16$ \\
3 & $y_{1}, y_{2}$ & $u_{2}, u_{3}$ & 13.34 & 21.80 \\
4 & $y_{1}, y_{3}$ & $u_{1}, u_{2}$ & 39.16 & 90.80 \\
5 & $y_{1}, y_{3}$ & $u_{1}, u_{3}$ & 2.84 & 2.54 \\
6 & $y_{1}, y_{3}$ & $u_{2}, u_{3}$ & 12.08 & 12.87 \\
7 & $y_{2}, y_{3}$ & $u_{1}, u_{2}$ & 38.28 & 88.69 \\
8 & $y_{2}, y_{3}$ & $u_{1}, u_{3}$ & $1.4 \mathrm{e}+16$ & $2.7 \mathrm{e}+15$ \\
9 & $y_{2}, y_{3}$ & $u_{2}, u_{3}$ & 11.80 & 12.76 \\
\hline
\end{tabular}

TABLE II: SVA 1 - systematic cancelling

engineering perspective. Thus, the pairing $u_{4} \leftrightarrow y_{2}, u_{2} \leftrightarrow y_{1}$ and $u_{3} \leftrightarrow y_{3}$ is chosen for this case.

\begin{tabular}{|c|c|c|c|c|}
\hline config & controlled & manipulated & \multicolumn{2}{|c|}{$\kappa\left(K_{e, n}\right), n=10,11,12$} \\
& variables & variables & OP 1 & OP 2 \\
\hline 10 & $y_{1}, y_{2}, y_{3}$ & $u_{1}, u_{2}, u_{4}$ & $6.3 \mathrm{e}+17$ & $2.2 \mathrm{e}+17$ \\
11 & $y_{1}, y_{2}, y_{3}$ & $u_{1}, u_{3}, u_{4}$ & $2.1 \mathrm{e}+16$ & $1.5 \mathrm{e}+16$ \\
12 & $y_{1}, y_{2}, y_{3}$ & $u_{2}, u_{3}, u_{4}$ & 19.06 & 18.10 \\
\hline
\end{tabular}

TABLE III: SVA 2 - systematic replacing

\section{SIMULATIVE VERIFICATION}

The recommended actions, which result from the previous analyses, are tested in simulation experiments (exps) and compared to the implemented control concept, s. Fig. 3.

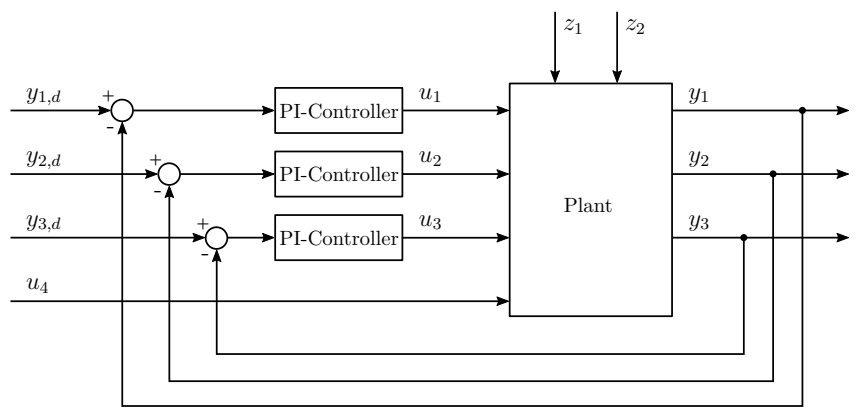

Fig. 3: Implemented control concept 
For simulations (s. Fig. 4), the plant model described in (13) is used. The recommended actions are briefly summarized as follows:

1) retaining the implemented concept (RGA), s. Fig. 4a,

2) interchange of $u_{1}$ and $u_{2}$ (DRGA), s. Fig. $4 \mathrm{~b}$,

3) omitting the third control loop (SVA 1), s. Fig. 4c,

4) manipulating $u_{4}$ instead of $u_{1}$ (SVA 2), s. Fig. 4d;

On the one hand, in exps 1) to 3), the same controller parameters are applied and the reference signal depends explicitly on $x_{6}$, such that

$$
y_{2, d}(t)=\left\{\begin{array}{cl}
y_{2, d, \text { max }} \cdot x_{6}^{2}(t), & y_{2, d}(t) \leq y_{2, d, \text { max }} \\
y_{2, d, \text { max }}, & \text { else }
\end{array}\right.
$$

with $y_{2, d, \max }=400 \mathrm{mbar}$. On the other hand, in exp 4), the controller parameters are adapted since a different physical quantity is manipulated. Additionally, a ramp, which does not depend on $x_{6}$, is chosen for $y_{2, d}$, as it would otherwise depend on the manipulated variable $u_{4}$. Hence, the simulation results for exps 1) to 3) can be compared directly. By comparing the results for exps 1) and 2), it turns out that an interchange of $u_{1}$ and $u_{2}$, improves the tracking and the disturbance behaviour of $y_{2}$, while the reactions of $y_{1}$ and $y_{3}$ are similar. A possible reason for this issue is that higher frequency domains are excited by the valve actuators' time delays, s. Sec. III-C. Since the performance declines for exp 3 ), an open third loop is not recommended. If $y_{2, d}$ may be independent of $x_{6}$, it seems convenient to manipulate $u_{4}$ instead of $u_{1}$ (exp. 4)). Moreover, it is possible to save energy by applying the latter approach as the blower is not driven at maximum rotational speed.

\section{CONCLUSIONS}

Based on the results of RGA, DRGA and SVA applied to a dynamic model of the product gas stage in a pyrolysis process, we derived recommendations to adapt the implemented control concept. Especially, DRGA revealed a modified pairing, if the system operates at higher frequency domains. With the help of a simplified calculation, we have shown that higher frequency domains can be excited due to the valve actuators' time delays. Numerical simulations confirm the improved performance of the closed loop when applying the modified pairing from DRGA. This modification has also been implemented successfully in the real plant. Hence, it is possible to overcome complicated disturbing effects, while retaining a simple control structure, which commonly leads to a higher acceptance by the plant personnel.

\section{ACKNOWLEDGMENT}

The authors thank their colleagues, Jerzy Depta and Oliver Scherer, for their support, proofreading and helpful advice.

\section{REFERENCES}

[1] D. E. Seborg, D. A. Mellichamp, T. F. Edgar, and F. J. Doyle III, Process dynamics and control. John Wiley \& Sons, 2010.

[2] W. S. Levine, The Control Handbook. CRC Press, Boca Raton, 1996, vol. 1.

[3] R. Osypiuk, B. Finkemeyer, and F. M. Wahl, "Multi-loop model-based control structure for robot manipulators," Robotica, vol. 23, no. 4, pp. 491-499, 2005.
[4] K. Weischedel and T. McAvoy, "Feasibility of decoupling in conventionally controlled distillation columns," Industrial \& Engineering Chemistry Fundamentals, vol. 19, no. 4, pp. 379-384, 1980.

[5] F. G. Shinskey, Process-control Systems: Application, Design, Adjustment 2nd Ed. McGraw-Hill, 1979.

[6] L. Tung and T. Edgar, "Analysis of control-output interactions in dynamic systems," AIChE Journal, vol. 27, no. 4, pp. 690-693, 1981.

[7] E. Bristol, "On a new measure of interaction for multivariable process control," IEEE transactions on automatic control, vol. 11, no. 1, pp. 133-134, 1966.

[8] M. Witcher, "Interacting control systems: steady state and dynamic measurement of interaction," ISA Trans., vol. 16, pp. 35-41, 1977.

[9] S. Skogestad and I. Postlethwaite, Multivariable feedback control: analysis and design. Wiley New York, 2007, vol. 2.

[10] J. Lunze, Regelungstechnik 2: Mehrgrößensysteme, Digitale Regelung Springer-Verlag, 2013.

[11] C. Smith, C. Moore, and D. Bruns, "A structural framework for multivariable control applications," in Proceeding of the Joint American Control Conference, Charlottesville, Virginia, 1981.

[12] J. Chen, J. S. Freudenberg, and C. N. Nett, "The role of the condition number and the relative gain array in robustness analysis," Automatica, vol. 30, no. 6, pp. 1029 - 1035, 1994. [Online]. Available: http://www.sciencedirect.com/science/article/pii/000510989490197X

[13] D. Chen and D. E. Seborg, "Relative gain array analysis for uncertain process models," AIChE journal, vol. 48, no. 2, pp. 302-310, 2002.

[14] J. P. Gagnepain and D. E. Seborg, "Analysis of process interactions with applications to multiloop control system design," Industrial \& engineering chemistry process design and development, vol. 21, no. 1 , pp. 5-11, 1982

[15] B. Wittenmark and M. E. Salgado, "Hankel-norm based interaction measure for input-output pairing," in Proc. of the 2002 IFAC World Congress, vol. 139, 2002.

[16] Q. Xiong, W.-J. Cai, M.-J. He, and M. He, "Decentralized control system design for multivariable processes a novel method based on effective relative gain array," Industrial \& engineering chemistry research, vol. 45, no. 8, pp. 2769-2776, 2006.

[17] H. Jiang, M. Wei, F. Xu, and X. Luo, "A dynamic relative gain array based on model predictive control," in Proceedings of the 10th World Congress on Intelligent Control and Automation, July 2012, pp. 33403344.

[18] F. Shinskey, "The stability of interacting control loops with and without decoupling," IFAC Proceedings Volumes, vol. 10, no. 6, pp. 21-30, 1977

[19] A. Jafarey, T. J. McAvoy, and J. M. Douglas, "Analytical relationships for the relative gain for distillation control," Industrial \& Engineering Chemistry Fundamentals, vol. 18, no. 2, pp. 181-187, 1979.

[20] T. McAvoy, "Interacting control-systems-steady-state treatment of dual composition control in distillation-columns," ISA TRANSACTIONS, vol. 16, no. 4, pp. 83-90, 1977.

[21] F. Shinskey, "Predict distillation column response using relative gains," Hydrocarbon Processing, vol. 60, no. 5, pp. 196-200, 1981.

[22] A. Papadourakis, M. F. Doherty, and J. M. Douglas, "Relative gain array for units in plants with recycle," Industrial \& engineering chemistry research, vol. 26, no. 6, pp. 1259-1262, 1987.

[23] T. McAvoy and N. Ye, "Base control for the tennessee eastman problem," Computers \& Chemical Engineering, vol. 18, no. 5, pp. 383-413, 1994

[24] C. Moore, "Application of singular value decomposition to the design, analysis, and control of industrial processes," in American Control Conference, 1986. IEEE, 1986, pp. 643-650.

[25] J. E. Rijnsdorp, "Interaction in two-variable control systems for distillation columns-ii," Automatica (Journal of IFAC), vol. 3, no. 1, pp. 29-52, 1965.

[26] H. Sigloch, Strömungsmaschinen, 3rd ed. Carl Hanser Verlag, 2006.

[27] R. Isermann, Mechatronic systems: fundamentals. Springer Science \& Business Media, 2007

[28] W. Wagner, Regel- und Sicherheitsarmaturen, 1st ed., ser. Vogel Fachbuch, Kamprath-Reihe. Vogel Buchverlag, 2008.

[29] G. Cerbe and G. Wilhelms, Technische Thermodynamik, 16th ed. Carl Hanser Verlag, 2011.

[30] R. Lamour, R. März, and C. Tischendorf, Differential-algebraic equations: a projector based analysis. Springer Science \& Business Media, 2013. 

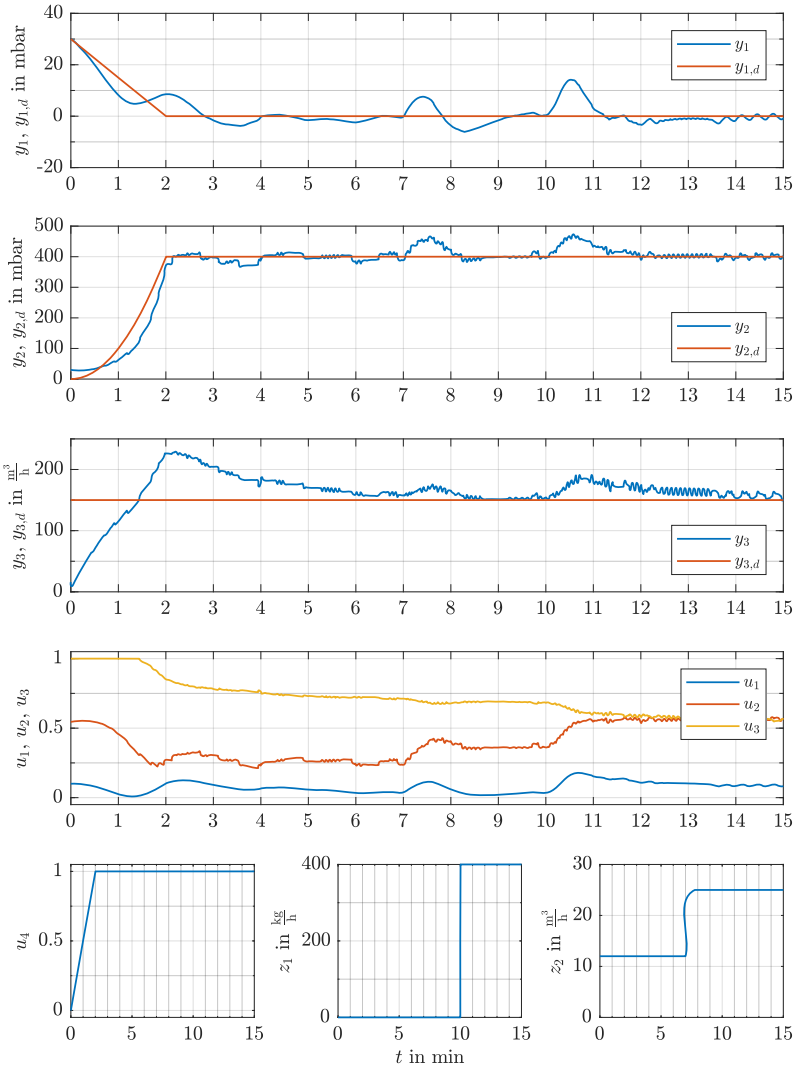

(a) Simulation with current concept (RGA)
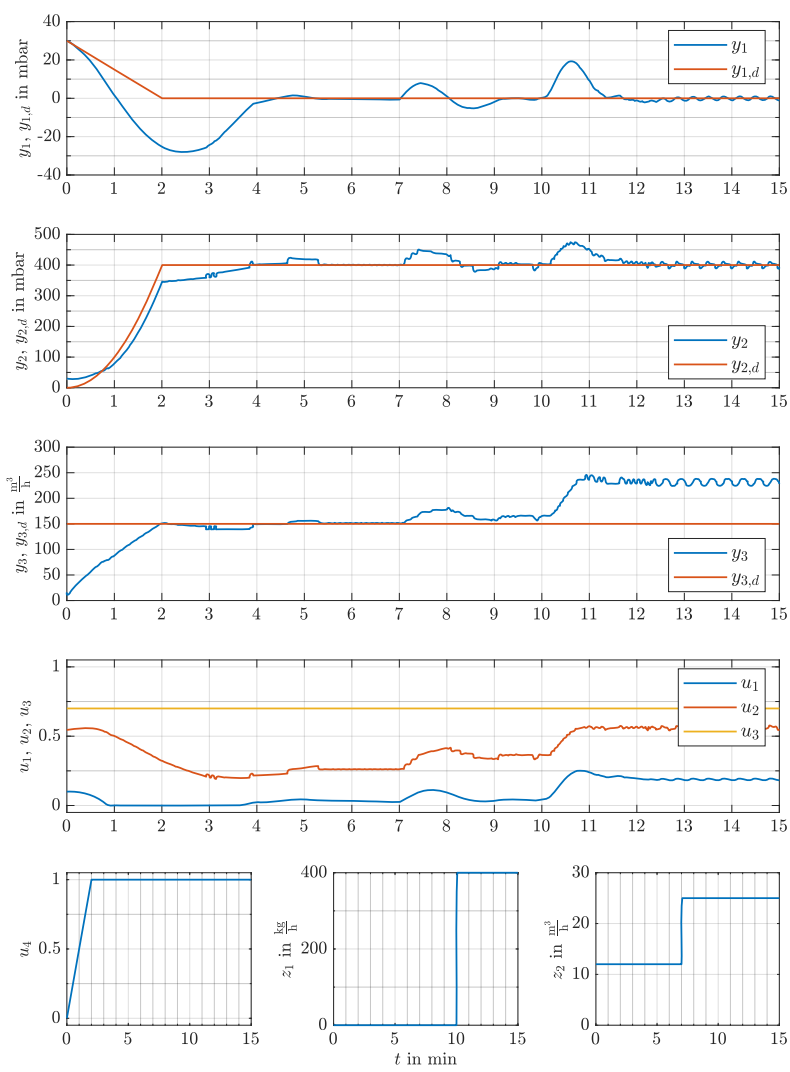

(c) Simulation with open third loop (SVA 1)
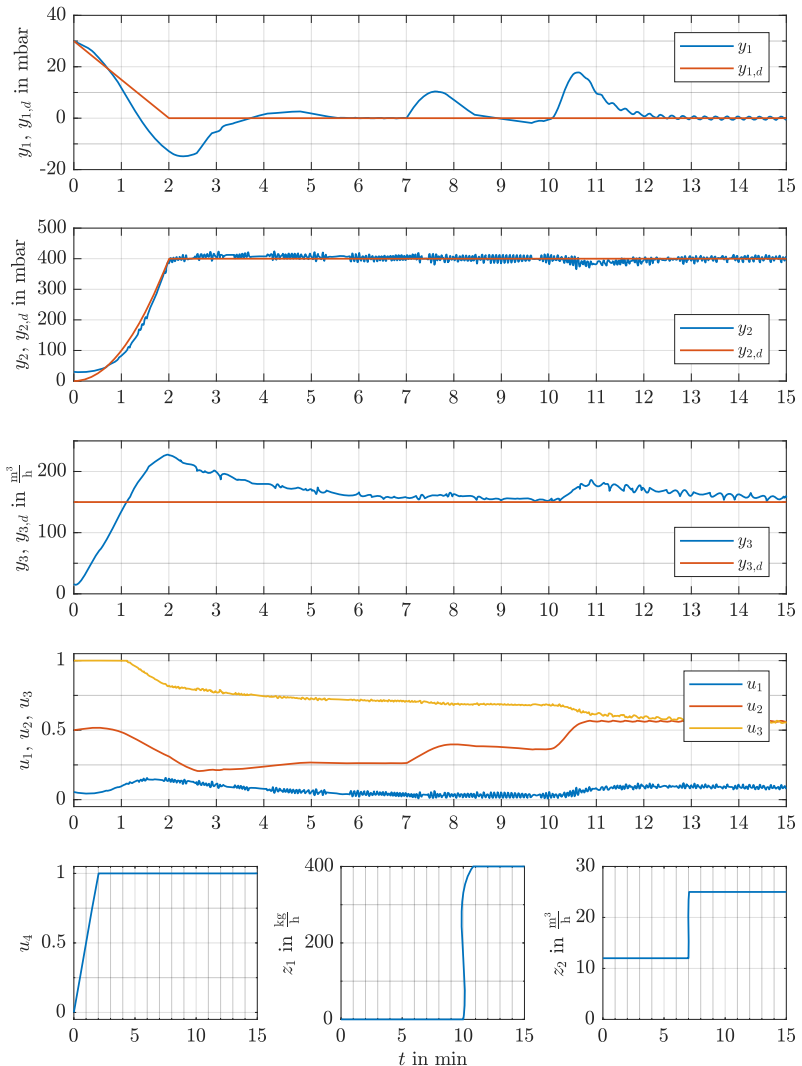

(b) Simulation with interchanged $u_{1}$ and $u_{2}$ (DRGA)
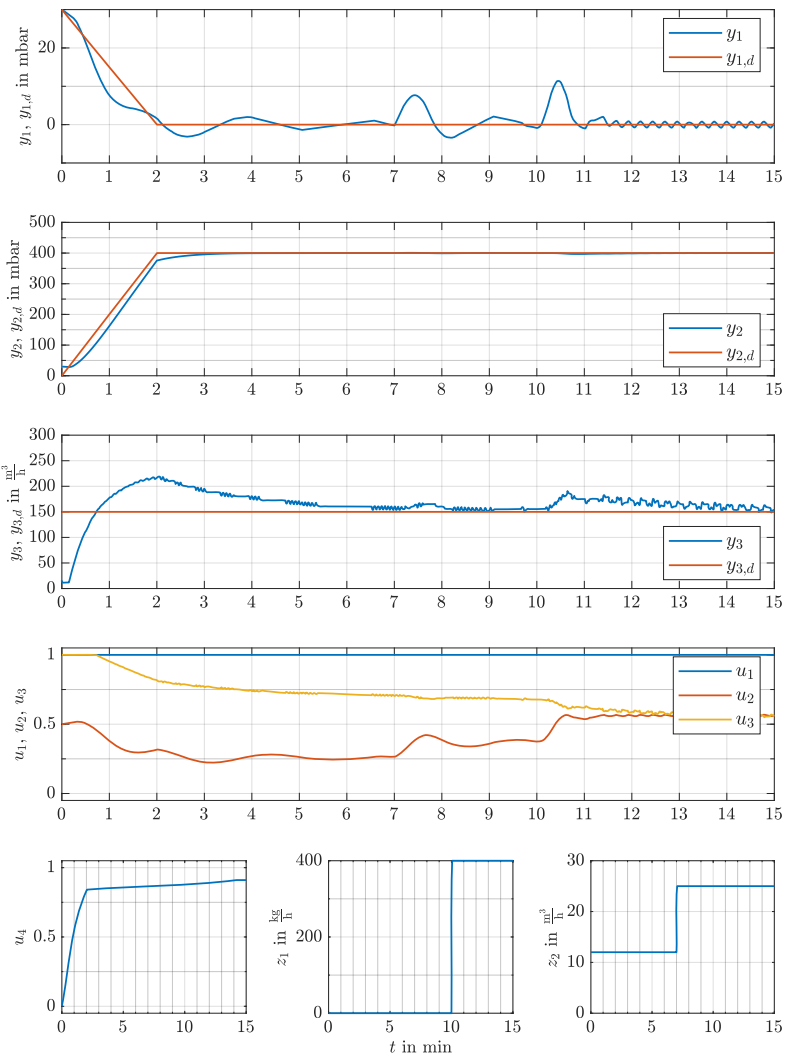

(d) Simulation for manipulating $u_{4}$ instead of $u_{1}$ (SVA 2)

Fig. 4: Simulation exps for results of RGA, DRGA, SVA 1 and SVA 2 